\title{
2 Familiengründung und unerfüllter Kinderwunsch bei Männern
}

\author{
Yve Stöbel-Richter und Elmar Brähler
}

\subsection{Braucht man(n) heutzutage noch eine Familie?}

Der gesellschaftliche Wandel in den letzten Jahrzehnten mit seinem rasanten Tempo eines Informationszeitalters, in welchem Erfindungen von heute Morgen schon „Schnee von gestern“ sind, greift auch massiv in die individuellen Lebensplanungen und -verläufe ein und hat nicht zuletzt auch das Bild von Männlichkeit stark infrage gestellt. Was braucht es zur Männlichkeit? Das Zeugen eines Sohnes, den Bau eines Hauses, das Pflanzen eines Baumes? Oder vielmehr ständige Flexibilität, Bereitschaft zum Risiko und eine körperliche und seelische Belastbarkeit, die über alle Grenzen erhaben scheint? Wenn denn eher letzteres die Antwort wäre, so ergibt sich hieraus eine fast unüberwindliche Diskrepanz zu Werten, auf denen eine Familiengründung basiert: Beständigkeit, Verlässlichkeit, Sicherheit.

Die Entscheidung, eine Familie zu gründen, unterliegt einem Prozess, welcher von anderen Lebensoptionen bestimmt wird: das Vorhandensein der „richtigen“ Partnerin, aber auch finanzielle und berufliche Sicherheit. Im Lebensverlauf gibt es daher zunächst Zeiträume, in denen eine Familiengründung nicht geplant und Kinderlosigkeit zunächst gewollt ist. Schwierig wird es dann, wenn sich die Familiengründung der Planung entzieht und aus der zunächst gewollten eine ungewollte Kinderlosigkeit resultiert.

War Elternschaft früher selbstverständlich, so wird heute mehr und mehr ein Problem daraus. Dabei sind Zögern, Abwägen und Aufschub kein privater Konflikt, sondern vielmehr Ausdruck des oben geschilderten gesellschaftlichen Wandels. Dieser Wandel hat dazu geführt, dass alte Bindungen aufgelöst wurden und neue Formen des Lebenslaufs und - damit einhergehend - neue Erwartungen und Anforderungen, neue Freiräume, und aber auch Abhängigkeiten entstehen. Vielfache Wahloptionen führen dazu, dass auch die Entscheidung, keine Familie zu gründen bzw. kinderlos zu bleiben, mehr und mehr zur gesellschaftlich anerkannten Lebensalternative geworden ist. 
Das folgende Kapitel setzt sich mit Fragen der Familiengründung auseinander - welche Motive sind ausschlaggebend, welche Bedingungen notwendig und fokussiert als spezielles medizinisches Problem - die ungewollte Kinderlosigkeit.

\subsection{Epidemiologie - Familiengründung bei Männern}

Im Jahr „2008 wurden in Deutschland 675.200 Kinder geboren. Dies war gegenüber dem Vorjahr ein Rückgang um 7.500 Geburten und lag nur geringfügig über dem historischen Tiefstand von 672.700 Geburten im Jahr 2006. Aus der absoluten Geburtenzahl für das Jahr 2008 ergibt sich eine Geburtenrate von 8,2 Kindern je 1.00o Einwohner. Der seit längerem zu beobachtende Geburtenrückgang erklärt sich vor allem aus einer sinkenden Zahl von Frauen im gebärfähigen Alter (15 bis 44 Jahre), denn die kopfstarken Altersgruppen der Babyboomerverlassen langsam aber sicher das Alter, in dem man überhaupt Kinder bekommen kann“ (Sievert u. Klingholz 2009, 4).

Amtliche Statistiken weisen zwar den Anteil kinderloser Frauen aus, aber über kinderlose Männer oder aber Väter liegen bis dato kaum Informationen vor.

Entgegen der Annahme, Männer würden, aufgrund ihrer längeren biologischen Zeugungsfähigkeit auch häufiger (sehr) spät Vater werden, zeigen doch Studienergebnisse, dass vor allem die erste Vaterschaft jenseits des 45. Lebensjahres ein eher seltenes Phänomen ist. Dies ist vor allem zwei Ursachen zuzuschreiben: einerseits nimmt auch bei Männern die Fruchtbarkeit im Alter ab, andererseits haben die meisten Männer eine nur wenige Jahre jüngere Partnerin, sodass auch hierdurch die Konzeptionsoptionen begrenzt werden. Anhand der Daten des Sozioökonomischen Panel aus dem Jahr 2001 (SOEP) identifiziert Schmitt (2004) für die Altersgruppe der 20- bis 45-Jährigen $53,6 \%$ als kinderlos, in der Altersgruppe der über 45-Jährigen 15,8\%. Grundsätzlich erfolgt der Übergang zur Elternschaft bei Männern im Vergleich zu Frauen verzögert, was auch damit zu tun hat, dass von vielen eine Vaterschaft nach wie vor erst nach erfolgreicher beruflicher Etablierung in Betracht gezogen wird.

Hank (2010) untersucht ebenfalls anhand der Daten des SOEP den Zusammenhang zwischen Elternschaft und Gesundheit. Die Ergebnisse zeigen, dass es bei Vätern einen positiven Zusammenhang zwischen Bildung und gesundheitlichem physischen Wohlbefinden gibt. Mütter und Väter mit vier und mehr Kindern schätzen ihre Gesundheit besser ein, als Mütter und Väter mit zwei Kindern. Der Zeitpunkt des Übergangs zur Elternschaft erweist sich allerdings für den Gesundheitszustand als irrelevant.

\subsection{Forschungsstand - Familiengründung bei Männern}

Aktuelle Forschungsarbeiten zur Familiengründung fokussieren nach wie vor sehr stark die weibliche Perspektive oder den Entscheidungsprozess auf Paarebene. Allerdings gibt es einige wenige Arbeiten, die die Familiengründung speziell bei Männern untersucht haben.

In der Studie „männer leben“(Helfferich et al. 2005) wurden im Zeitraum 2001-2004 reproduktive Biographien von Männern zwischen 25 und 54 Jahren näher analysiert. Die Ergebnisse aus Interviews und Fragebogenerhebung zeigen bei den Männern eine 
überwiegend starke Partnerschaftsorientierung; 62 Prozent sind verheiratet, zwei Drittel haben eigene Kinder. Bei den höher qualifizierten Männern zeigt sich ein deutliches Aufschieben des Übergangs zur ersten Elternschaft. Darüber hinaus ist ein Zusammenhang zwischen Einkommen und Bindungsverhalten nachweisbar: Je höher das Einkommen, desto größer die Wahrscheinlichkeit, dass die Männer mit einer Partnerin zusammen leben und auch gemeinsame Kinder haben; Männer mit niedrigem Einkommen sind häufiger Single. Als ebenfalls einkommensabhängig erweist sich die gewünschte und realisierte Kinderzahl. Kinderlosigkeit ist am häufigsten auf eine fehlende Partnerin zurückzuführen, dabei wird allerdings nicht zwischen gewollter und ungewollter Kinderlosigkeit unterschieden. Hinsichtlich des Übergangs zur Vaterschaft zeigt sich ein „Zeitfenster“ zwischen zu jung und zu alt, dabei liegt die mittlere angegebene Altersgrenze bei 5o,5 Jahren. Mit zunehmendem Alter lässt der Wunsch nach weiteren Kindern nach.

In einer Auswertung des Familiy and Fertility Survey (FFS) stellten Eckardt und Klein (2004; 2006) für die Männer der Stichprobe fest, dass oftmals der Kinderwunsch erst vor dem Hintergrund einer konkreten Paarbeziehung entsteht und vom Alter der Partnerin abhängig ist. Männer mit höherem Schulabschluss geben einen stärkeren Wunsch nach einer Familiengründung an. Arbeitslosigkeit wirkt sich bei Männern negativ auf den Wunsch zur Elternschaft aus, bei Frauen hingegen tendenziell positiv. Auch hinsichtlich des Einkommens sind gegenläufige Effekte auf den Kinderwunsch von Männern und Frauen ersichtlich: Der Elternschaftswunsch sowie auch der Wunsch zur Familienerweiterung nimmt bei Männern mit der Höhe des Einkommens zu. Weitere Ergebnisse deuten darauf hin, dass ein höheres Einkommen der Männer nicht nur deren eigenen Elternschaftswunsch, sondern auch den ihrer Partnerinnen positiv beeinflusst. Für Männer ist der Wunsch zur Elternschaft stark von der Einschätzung der aktuellen Partnerschaft als stabile Beziehung abhängig.

Schmitt (2004) kommt in seiner Analyse von Daten aus dem Sozioökonomischen Panel (SOEP; $\mathrm{n}=8.639$ Personen) zu folgenden Ergebnissen: In den Altersgruppen zwischen 20 und 65 Jahren sind im Vergleich zu Frauen mehr Männer kinderlos. Besonders auffällig ist die Altersgruppe der 35- bis 40-Jährigen: in dieser sind 36,4\% Männer kinderlos im Vergleich zu 19,1\% der Frauen. Schmitt identifizierte zwei Cruppen von Männern, die häufig kinderlos sind: erstens Männer ohne Schulabschluss und mit sehr niedrigem Einkommen und zweitens Männer mit Abitur, aber ohne Studium. Der mit 29,6\% besonders hohe Anteil an Kinderlosen unter 45-Jährigen mit Abitur wird in der Studie auf eine starke Berufsorientierung dieser Gruppe zurückgeführt. Demnach zeugen „Durchschnittsverdiener“ am ehesten Kinder, ab einem Nettojahresgehalt von 20.0oo Euro steigt auch die Kinderlosigkeit an. So bleiben etwa ein Viertel der Männer wie der Frauen in der oberen Einkommensklasse (ab 30.000 Euro) dauerhaft kinderlos.

In der Sächsischen Längsschnittstudie (Stöbel-Richter 2010) zeigt sich, dass der Auszug aus dem Elternhaus bei den Männern später als bei den Frauen erfolgt, aber in keinem direkten Zusammenhang mit der eigenen Familiengründung steht. Auch in dieser Stichprobe entscheiden sich Männer später für eine Familiengründung als Frauen und sind im Alter von Mitte 30 noch häufiger kinderlos. Männer und Frauen unterscheiden sich nur geringfügig hinsichtlich ihrer Einstellungen zum Kinderwunsch bzw. in den Motiven zur Familiengründung; Männer geben stärkere Ängste vor persönlichen Einschränkungen und einen stärkeren Wunsch nach sozialer An- 
erkennung durch ein Kind an. Als deutlicher Einflussfaktor auf Familiengründung erweist sich die Erfahrung von Arbeitslosigkeit; je länger diese andauert, desto eher wird die Gründung einer Familie aufgeschoben.

Nach der idealen Lebensform befragt, suchen Männer (87\%) und Frauen (91\%) überwiegend „einen Raum geprägt von Stabilität und Liebe“ für das Gelingen des eigenen Lebens. Mehr Männer (17\%) als Frauen (13\%) wollen dies ohne Kinder, aber weitaus mehr für ihre Kinder (Männer 70\%, Frauen 77\%). Lediglich 5\% der Männer (2\% der Frauen) geben „kurze Partnerbeziehungen“ als für sie ideale Beziehungsform an (Volz u . Zulehner 2009).

Das Aufschieben der Erstelternschaft und übersteigerte Erwartungen an die Reproduktionsmedizin, einen unerfüllten Kinderwunsch auch in späteren Lebensjahren noch erfolgreich behandeln zu können, führt letztendlich zu einer Erhöhung der Risiken für eine Verminderung der Fekundität (Glander 2005).

Der Weg in die Kinderlosigkeit - so die Ergebnisse des Bamberger-Ehepaar-Panels ist oftmals geprägt durch Hürden in Form von Voraussetzungen und Befürchtungen. Bezogen auf eine gewollte oder ungewollte Kinderlosigkeit zeigen die Ergebnisse weniger klare Entscheidungsprozesse und somit einander ausschließende Kategorien, sondern vielmehr ein Kontinuum zwischen familienfreundlichen und familienkritischen Einstellungen (Rupp 2005).

Betrachtet man die Familiengründung bei Männern, so zeigt sich ein deutlicher Aufschub dieser Entscheidung aufgrund verschiedener Aspekte, wie z.B. ein später Auszug aus dem Elternhaus und eine durchschnittlich 3 Jahre jüngere Partnerin. Zusätzlich zeigt sich ein deutlicher Zusammenhang mit der Einkommenssituation und dem Partnerschaftsstatus. In der Kohortenfolge zeigen sich ein immer deutlicherer Aufschub der Familiengründung und eine steigende Prävalenz dauerhafter Kinderlosigkeit. Hinsichtlich des Partnerschaftsstatus zeigt sich, dass unter den Kinderlosen, kurz vor Abschluss der fertilen Phase, etwa zwei Drittel der Männer nicht in einer Partnerschaft leben (Schmidt u. Winkelmann 2005).

\subsection{Ungewollte Kinderlosigkeit bei Männern}

Zahlen zur ungewollten Kinderlosigkeit sind nur schwer aus verlässlichen Statistiken ableitbar, da in diesen nicht zwischen gewollter und ungewollter Kinderlosigkeit unterschieden wird. In der Reproduktionsmedizin ist lange Zeit der Mann als Ursache für eine ungewollte Kinderlosigkeit überhaupt nicht in Betracht gezogen worden. Inzwischen weiß man aber, dass ca. ein Drittel bis die Hälfte der Ursachen für ungewollte Kinderlosigkeit auf männliche Infertilität zurückgeführt werden können (Könnecke u. Wilke 2002; Glander 2005).

\footnotetext{
„Mit ungewollter Kinderlosigkeit wird ein Zustand bezeichnet, der durch Leiden an einer Unfruchtbarkeit (auch als Infertilität bzw. Sterilität bezeichnet) gekennzeichnet ist. 1967 wurde die ungewollte Kinderlosigkeit (Zeugungs- und/oder Empfängnisunfähigkeit) durch die Scientific Group on the Epidemiology of Infertility der WHO als Krankheit anerkannt “(Strauß u. Beyer 2004, 7).
} 
„Der WHO-Definition entsprechend ist eine Infertilität/Sterilität zu diagnostizieren, wenn bei einem Paar entgegen seinem expliziten Willen nach mehr als 24 Monaten trotz regelmäßigem, ungeschütztem Sexualverkehr keine Schwangerschaft eintritt (ICD-10 Diagnosen: Sterilität der Frau [N97.x], männliche Sterilität [N46]).

Bei der Entstehung, dem Verlauf und der Therapie der ungewollten Kinderlosigkeit spielen sowohl biologische, psychologische als auch soziale Faktoren eine Rolle“" (Strauß u. Beyer 2004, 7). In der Praxis findet allerdings häufig eine Behandlung schon nach einem Jahr ungewollter Kinderlosigkeit statt (Rowe et al. 2000; Nieschlag 2009).

Zahlen zur Prävalenzrate sind sehr unterschiedlich und auch unterschiedlicher Datenlagen geschuldet. Aus bevölkerungsrepräsentativen Querschnittserhebungen weiß man, dass die Zahl der dauerhaft ungewollt kinderlosen Menschen sich auf ca. $3 \%$ beläuft. Damit sind frühere Schätzungen, welche von 10-15\% ausgingen, zu hoch gegriffen. Allerdings ist auch nachgewiesen, dass 20-30\% aller Paare einmal in ihrem Leben unter verminderter Fruchtbarkeit leiden (Ittner et al. 20oo, Brähler u. StöbelRichter 2002) und 6-9\% aller Paare in Mitteleuropa ungewollt kinderlos sind und eine Behandlung wünschen. 30\% aller Frauen erleben im Verlauf ihrer reproduktiven Lebensphase eine mindestens 12-monatige Episode der Unfruchtbarkeit (ESHRE Capri Workshop 2001).

\footnotetext{
In ihrem Review beschreiben Boivin et al. (2007) eine weltweite mediane Prävalenzrate für Sterilität, also dem Ausbleiben einer Schwangerschaft trotz regelmäßigem, ungeschütztem Geschlechtsverkehr über einen Zeitraum von 12 Monaten, von 9\% (Range 5-15\%). Die in der vorhandenen Literatur sehr variablen Angaben zur Häufigkeit von Infertilität scheinen zum Großteil auf Unterschiede in der Definition zurückzuführen zu sein (Greenhall u. Vessey 1990; Kentenich 2010).
}

Häufigste Sterilitätsursache beim Mann ist eine eingeschränkte Spermienqualität oder Störungen der Spermatogenese. Weitere Ursachen können urogenitale Auffälligkeiten oder funktionelle Störungen (erektile Dysfunktion oder Ejakulationsstörungen) sein. Grundsätzlich wird bei Vorliegen eines unerfüllten Kinderwunsches eine umfassende andrologisch-klinische Untersuchung mit Untersuchung des Genitales empfohlen (Strauß et al. 2004; Strauß u. Beyer 2004; Glander 2005) (s. Kap. II.1). Bei ca. einem Viertel der von Infertilität betroffenen Männer bleibt allerdings die ursprüngliche Infertilitätsursache ungeklärt (Glander 2005).

Ein unerfüllter Kinderwunsch ist für das Paar und somit auch für den Mann ein Stressor, der zu den stressvollsten Lebenssituationen überhaupt gehören kann - vergleichbar mit dem Verlust eines Partners oder dem Tod eines Kindes. Die emotionalen Reaktionen auf die Diagnose „Fertilitätsstörung“ reichen von Schock und Erstaunen bis hin zu Depression und Trauer (Spiewak 2002; vgl. auch Freeman et al. 1985; Burns u. Covington 1999; Carl 2002; Kowalcek et al. 2007; Beyer et al. 2004).

Für Männer geht die Diagnose oft mit einer Erschütterung des Selbstwertgefühls, narzisstischer Kränkung und der Erfahrung von Hilflosigkeit einher, die mitunter 
in Trauer und Depression münden können (Könnecke u. Wilke 2002; Connolly et al. 1992). Die Kausalität zwischen Infertilität und Partnerschaftsproblemen kann in beide Richtungen nachgewiesen werden; einerseits entstehen Partnerschaftsprobleme (oft auf sexueller Ebene) auf dem mitunter sehr langen Leidensweg des unerfüllten Kinderwunsches, andererseits können diese aber auch aus Behandlungsmisserfolgen nach einer reproduktionsmedizinischen Behandlung resultieren. Darüber hinaus berichten verschiedene Studien unterschiedliche Partnerschaftszufriedenheiten in Abhängigkeit von der Sterilitätsursache (vgl. Könnecke u. Wilke 2002).

Betrachtet man kinderlose Paare allerdings langfristig, so können überwiegend keine Unterschiede zu Personen mit Kindern und keine Beeinträchtigungen durch Einsamkeit trotz weniger umfassender sozialer Netzwerke festgestellt werden (Beyer et al. 2004).

Aber auch eine erfolgreiche reproduktionsmedizinische Behandlung von ungewollter Kinderlosigkeit kann bei Männern zu einer stärkeren sozialen Isolierung führen, als bei Männern, in Partnerschaften mit Spontankonzeptionen (Küchenhoff $u$. Könnecke 2000).

Schätzungen gehen davon aus, dass in Deutschland ca. 3\% aller Kinder mit Hilfe reproduktionsmedizinischer Maßnahmen gezeugt werden (Strauß et al. 2004). Allerdings verlässt die Mehrzahl der Paare, die eine Kinderwunschbehandlung in Anspruch nehmen, diese auch wieder kinderlos; vor allem dann, wenn die Frauen bereits älter als 40 Jahre sind (Stöbel-Richter et al. 2010). Auch wenn Deutschland im europäischen Vergleich hinsichtlich der erfolgten Punktionen und klinischen Schwangerschaften nach Frankreich und Großbritannien auf dem dritten Platz liegt (vgl. DIR 2009), sind doch die endgültigen Erfolgsquoten eher ernüchternd. Für das Jahr 2008 lagen die klinischen Schwangerschaftsraten bei $28 \%$. Setzt man die Zahl der durchgeführten Behandlungen in Kontext zu den eingetretenen Geburten, so ergibt sich eine Erfolgsquote von 10,6\% (DIR 2009). In Abhängigkeit vom Alter liegen die klinischen Schwangerschaftsraten bzw. Raten des Embryotransfers bei mit IVF oder ICSI behandelten Frauen zwischen 30 und 34 Jahren bei 34,4\%, und lediglich bei 17,7\% bei Frauen über 40 Jahre (DIR 2009).

Eine ungewollte Kinderlosigkeit muss als dynamischer Prozess der lebenslangen Bewältigung betrachtet werden (Könnecke u. Wilke 2002). Der adäquate Umgang mit hilfesuchenden Paaren erfordert neben der medizinischen Behandlung eine psychosomatische Betrachtungsweise des Geschehens und die Integration psychischer, sozialer und familialer Aspekte in die behandlungsbegleitende Beratung.

\section{Literatur}

Beyer K, Gagel D, Bindt C, Felder H, Henning K, Goldschmidt S, Stöbel-Richter Y, Wischmann T, Stammer H, Verres R, Kentenich H, Strauß B, Brähler E (2004) Quellentext zur Leitlinie Psychosomatisch orientierte Diagnostik und Therapie bei Fertilitätsstörungen. 2. Belastungen durch ungewollte Kinderlosigkeit und weiterer Verlauf nach erfolgreicher reproduktionsmedizinischer Behandlung. In Strauß, B, Brähler E \& Kentenich H (Hrsg.) Fertilitätsstörungen - psychosomatisch orientierte Diagnostik und Therapie. Schattauer Stuttgart, 46-60

Boivin I, Bunting L, Collins JA, Nygren KG (2007) International estimates of infertility pravalence and treatmentseeking: potential need and demand for infertility medical care. Hum Reprod 22, 1506-1512

Brähler E, Stöbel-Richter Y (2002) Familienfeindlicher Zeitgeist? - Zum Wandel im Reproduktionsverhalten in Deutschland und im europäischen Vergleich. Reproduktionsmedizin 18, 276-282 
Burns LH, Covington SH (1999) (eds.) Infertility counceling. A comprehensive handbook for clinicans. Parthenon Publishing New York

Carl C (2002) Leben ohne Kinder. Wenn Frauen keine Mütter sein wollen. Rowohlt, Reinbek bei Hamburg

Connolly KH, Edelmann RI, Cooke ID, Robson I (1992) The impact of infertility on psychological functioning. I Psychosom Res 36, 459-468

DIR (2009) Deutsches IVF-Register. http://www.deutsches-ivf-register.de/pdf-downloads/dirjahrbuch2008.pdf, abgerufen am 15.06.2010

Eckard I, Klein T (2004) Kinderwunsch, Kinderzahl und Kinderlosigkeit von Männern. Projektbericht, unveröffentlicht

Eckard J, Klein T (2006) Männer, Kinderwunsch und generatives Verhalten. Schriften des DII: Familiensurvey Band 13. Deutsches Jugendinstitut D|I München

ESHRE Capri Workshop Group (2001) Social determinants of human reproduction. Human Reproduction 16, $1518-1526$

Freeman EW, Boxer AS, Rickels K (1985) Psychological evaluation and support in a program of in vitro fertilization and embryo transfer. Fertility and Sterility 43, 48-53

Glander H I (2005) Unerfüllter Kinderwunsch. In: Schill W-B, Bretzel RG, Weidner W (Hrsg.) MännerMedizin. Urban \& Fischer München, 168-172

Greenhall E, Vessey M (1990) The prevalence of subfertility: a review of the current confusion and a report of two new studies. Fertil Steril 54, 978-983

Hank K (2010) Childbearing history, later life health, and mortality in Germany. http://www.diw.de/soeppapers, abgerufen am 15.06.2010

Helfferich C, Klindworth H, Wunderlich H (2005) männer leben. Eine Studie zu Lebensverläufen und Familienplanung im Auftrag der BZgA. Köln: BZgA

Ittner E, Himmel W, Kochen MM (2000) Ungewollte Kinderlosigkeit in der Hausarztpraxis: Beratungs- und Betreuungsbedarf. In: Brähler E, Felder H, Strauß B (Hrsg.) Fruchtbarkeitsstörungen. Jahrbuch der Medizinischen Psychologie 17, 229-244. Hogrefe Göttingen

Kentenich H (2010) Fertilitätsstörungen - psychosomatisch orientierte Diagnostik und Therapie. Überarbeitete Version. Manuskriptfassung

Könnecke R, Wilke S (2002) Bewältigungsmuster bei ungewollt kinderlosen Männern. Reproduktionsmedizin 18, $73-82$

Kowalcek I, Rohde A, Kentenich H (2007) Psychologie des Kinderwunschpaares. In: Felberbaum RE, Bühler K, Ven H van der (Hrsg.) Das Deutsche IVF-Register 1996-2006 - 10 Jahre Reproduktionsmedizin in Deutschland. Springer Heidelberg, 157-166

Küchenhoff J, Könnecke R (2000) Der (unerfüllte) männliche Kinderwunsch und seine Bedingungen. In: Brähler E, Felder H, Strauß B (Hrsg.) Fruchtbarkeitsstörungen. Jahrbuch der Medizinischen Psychologie 17, 124-145, Hogrefe Göttingen

Nieschlag E (2009) Aufgaben und Ziele der Andrologie. In: Nieschlag E, Behre HM, Nieschlag S (eds.) Andrologie - Grundlagen und Klinik der reproduktiven Gesundheit des Mannes. 3. Auflage, Springer, Berlin, Heidelberg, New York, S. 4-12

Rowe PI, Comhaire FH, Hargreave TB, Mahmoud AMA (2000) WHO manual for the standardized investigation, diagnosis and management of the infertile male. Cambridge University Press, Cambridge

Rupp M (2005) Kinderlosigkeit in stabilen Ehen. Zeitschrift für Familienforschung 17, 21-40

Schmitt C (2004) Kinderlose Männer in Deutschland - Eine sozialstrukturelle Bestimmung auf der Basis des Sozioökonomischen Panels (SOEP). Berlin: Deutsches Institut für Wirtschaftsforschung (DIW)

Schmitt C, Winkelmann U (2005) Wer bleibt kinderlos? Sozialstrukturelle Daten zur Kinderlosigkeit von Frauen und Männern. DIW Berlin Discussion Papers 473, http://www.econstor.eu/bitstream/10419/18324/1/ dp473.pdf, abgerufen am 15.06.2010

Sievert S, Klingholz (2009) Ungleiche Nachbarn. Die demografische Entwicklung in Deutschland und Frankreich verläuft gegensätzlich - mit enormen Langzeitfolgen. Berlin-Institut für Bevölkerung und Entwicklung. http://www.berlin-institut.org/fileadmin/user_upload/Veroeffentlichungen/Frankreich/Ungleiche_Nachbarn_online_NEU.pdf, abgerufen am 15.05.2010

Spiewak M (2002) Wie weit gehen wir für ein Kind? Eichborn Frankfurt 
Stöbel-Richter Y (2010) Fertilität und Partnerschaft. Eine Längsschnittstudie zu Familienbildungsprozessen über 20 Jahre. Psychosozial-Verlag Gießen

Stöbel-Richter Y, Brähler E, Weidner K (2010) Chancen und Risiken moderner reproduktionsmedizinischer Verfahren. In: Remmers H, Kohlen H (Hrsg.) Bioethics, Care and Gender, 147-162, Universitätsverlag Osnabrück

Strauß B, Beyer K (2004). Ungewollte Kinderlosigkeit. Gesundheitsberichterstattung des Bundes (RKI). Band 20. http://edoc.rki.de/documents/rki_fv/reUzuR53|xg|l/PDF/27ZlDyKPODMF_5320.pdf, abgerufen am 15.05.2010

Strauß B, Brähler E, Kentenich H (2004) (Hrsg.) Fertilitätsstörungen - psychosomatisch orientierte Diagnostik und Therapie. Schattauer Stuttgart

Volz R, Zulehner PM (2009) Männer in Bewegung. Zehn Jahre Männerentwicklung in Deutschland. Forschungsreihe des BMFSFJ, Band 6. Baden-Baden: Nomos-Verlag. http://www.bmfsfj.de/RedaktionBMFSFJ/Broschuerenstelle/Pdf-Anlagen/M_C3_A4nner-in-Bewegung,property=pdf,bereich=bmfsfj,sprache=de,rwb=true.pdf, abgerufen am 15.05.2010 